\title{
Application of transrectal ultrasound in guiding interstitial brachytherapy for advanced cervical
}

\section{cancer}

\author{
Yuangiang Lin, MD', Dan Shi, MD², Hequn Li, MD', Guanghui Cheng, MD², Hui Wang, MD' \\ 'Department of Ultrasound, China-Japan Union Hospital, Jilin University, Changchun, China, ${ }^{2}$ Department of Radiotherapy, China-Japan Union \\ Hospital, Jilin University, Changchun, China
}

\begin{abstract}
Purpose: To investigate the role of transrectal ultrasound guidance in interstitial brachytherapy for cervical cancer.

Material and methods: Forty-eight patients who underwent interstitial brachytherapy treatment for cervical cancer between January 2017 and January 2018 were enrolled in the study. The distances between each inserted needle and the lesion were measured at seven sites by ultrasound (D1-D7) and compared to the corresponding distances (M1-M7) when visualised with nuclear magnetic resonance imaging (MRI). Measurements were paired on the basis of the observation sites, e.g. D1 and M1, D2 and M2. The statistical differences, intraclass correlation coefficients (ICCs), and linear relationships for the paired measurements were calculated.

Results: No significant differences were found between the paired M and D measurements, with all ICCs showing high levels of concordance (0.81-0.93).

Conclusions: Transrectal ultrasound showed strong agreement with MRI results in determining the position of the inserted needles. Transrectal ultrasound is a useful tool for guided interstitial brachytherapy and is appropriate for widespread use in the treatment of locally advanced cervical cancer.

J Contemp Brachytherapy 2020; 12, 4: 375-382 DOI: https://doi.org/10.5114/jcb.2020.98118
\end{abstract}

Key words: cervical cancer, transrectal ultrasound, brachytherapy, ultrasound guidance.

\section{Purpose}

Cervical cancer, one of the most common malignant tumours of the female reproductive system, has high morbidity and mortality [1]. Early cervical cancer is typically treated with surgery, while patients intolerant to surgery or with advanced cancer are often administered radiotherapy combined with chemotherapy [2]. Radiation treatment methods include external and brachytherapy; of these, brachytherapy has unique dosimetric advantages and plays a key role in radical treatment $[3,4]$. However, for patients with irregular tumour shapes or larger lesion ranges, simple intrauterine brachytherapy cannot meet the dosimetric requirements of radical radiotherapy, and close-range interstitial brachytherapy is needed $[5,6]$.

Interstitial brachytherapy is an invasive operation, and its most important consideration is to ensure the safety of the patient and accuracy of needle insertion $[7,8]$. Therefore, several image guidance methods have been proposed. Previous studies have confirmed that real-time ultrasound guidance during insertion can improve safety and reduce complications [9]. However, while the use abdominal ultrasound-guided insertion has been reported in many cases recently $[10,11]$, there are a lack of data regarding the safety and efficacy of transrectal methods. Abdominal ultrasound-guided needle insertion is susceptible to hindrance caused by factors such as intestinal gas, obesity, and posture, and images may be not clear enough for clinical use $[12,13]$. However, while transrectal ultrasound provides clearer images of the structure of the uterus and surrounding tissues and guides insertion of the needle, there are few reports in the literature on the accuracy of this process. In our hospital, interstitial brachytherapy of cervical cancer guided by abdominal ultrasound and transrectal ultrasound has been used for many years [14]. This study compared the accuracy of transrectal ultrasound-guided needle insertion to magnetic resonance imaging (MRI)-guided insertion in interstitial brachytherapy for locally advanced cervical cancer.

\section{Material and methods \\ Patient selection}

Forty-eight patients with cervical cancer treated in the radiation therapy department of our hospital be- 
tween January 2017 and January 2018 were recruited as subjects. The patients were between 35 and 72 years of age (average age $59 \pm 7$ years) and had been diagnosed with cervical squamous cell carcinoma ( 45 cases), cervical adenocarcinoma (one case), or cervical adenosquamous carcinoma (two cases). The disease stages were IIa2 (two patients), IIb (five patients), IIIa (two patients), and IIIb (39 patients).

The inclusion criteria were: 1) locally advanced cervical cancer with completed external irradiation treatment and non-obvious tumour regression, non-obvious parametrial infiltration regression, eccentric lesions, or irregular tumour shape after regression, 2) treatment with a Utrecht Applicator (NUC, Fernandal, Netherlands) and matching interstitial needles, and 3) MRI image localisation performed after the needles were inserted.

We excluded patients: 1) with severe heart and lung diseases, 2) with a history of hysterectomy, 3) for whom it was impossible to insert an intrauterine tube, 4) who could not undergo transrectal ultrasound, such as those with acute inflammation or injury around the rectum and anorectal stenosis, 5) who could not undergo an MRI examination, such as those with ferromagnetic implants in vivo or claustrophobia, and 6) who could not afford anaesthesia or treatment.

\section{Research methods}

A pre-treatment plan was made according to the condition of each patient. Patients fasted for 8-12 hours before the brachytherapy procedure and then underwent bowel
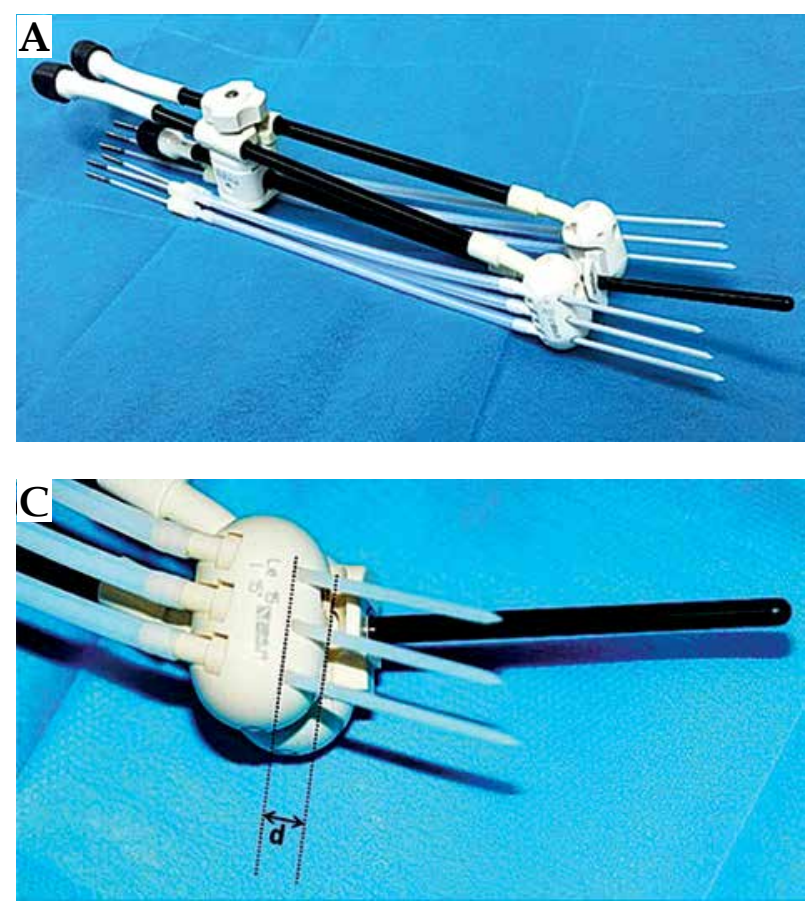

and preoperative preparation. After being placed under general anaesthesia, the patient was placed in the lithotomy position, and the uterus was probed under abdominal ultrasound guidance. All ultrasound images were recorded.

We used a CA541 abdominal probe (Esaote, Genoa, Italy) to calculate the angle of the uterine cavity. Based on this angle, a suitable intrauterine tube and the Utrecht applicator, with ovoids of different angles and interstitial needles (Figure 1A), were inserted and adapted to the vaginal angle. The angle $\theta$ between the interstitial needle and the intrauterine tube was equal to the difference between the angle of the intrauterine tube and the angle of the ovoids used in the patient (Figure $1 \mathrm{~B}$ ). " $\mathrm{D}$ " parameters were calculated as the distance between the insertion point of the interstitial needle and various surfaces of the ovoid (Figure 1C). The interstitial needle was subsequently guided by transrectal ultrasound with a TRT33 transrectal double-plane probe (Esaote, Genoa, Italy). In the insertion process, the interstitial needles were numbered according to the uniform order (Figure 1D). During the longitudinal scan, the probe was inserted about $10.0 \mathrm{~cm}$ into the rectum and the ultrasonic sweep depth was set to $6.0 \mathrm{~cm}$. Because of the long distance over which the probe entered the rectum, and the inability to place the probe parallel to the intrauterine tube due to the uterine angle, the scan was performed along the direction of the interstitial needles. During the insertion process, the angles and depth of the needles were adjusted with reference to the ultrasound and the pre-treatment plan. After insertion, the probe was withdrawn about $5.0 \mathrm{~cm}$ to perform transverse scanning of
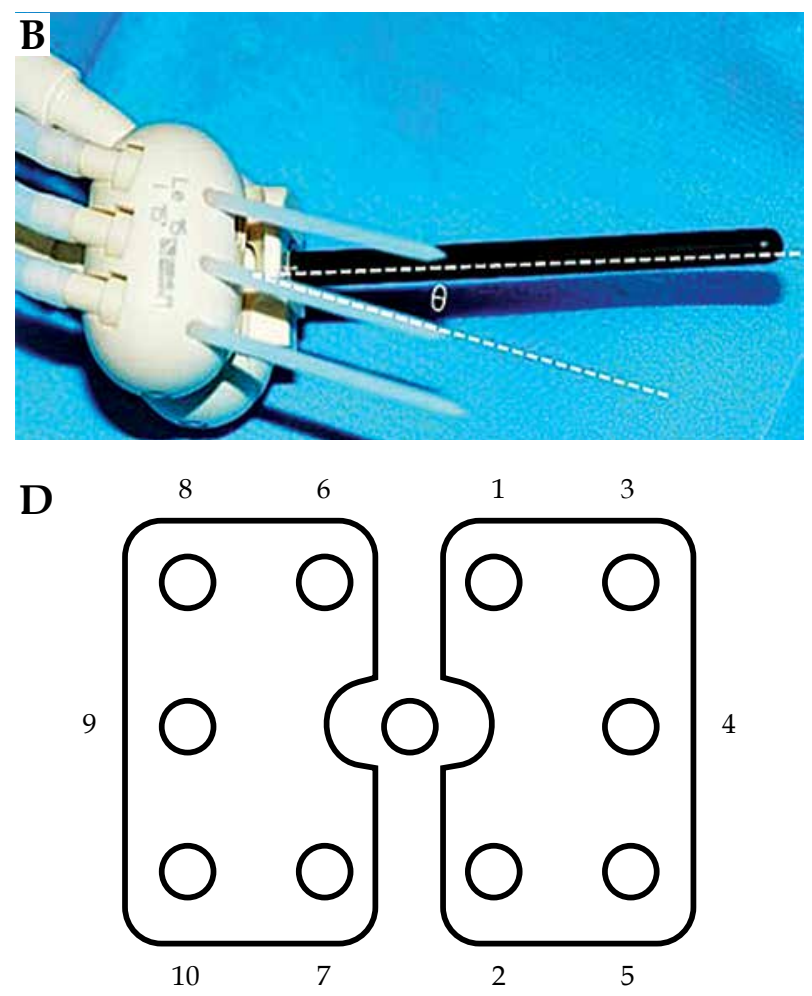

Fig. 1. Utrecht interstitial applicator. A) The Utrecht applicator and the matching interstitial needles, B) There is an angle $\theta$ between intrauterine tube and interstitial needle, $\theta=$ the angle of intrauterine tube - angle of ovoids, C) There is a distance " $\mathrm{d}$ " between the insertion point of interstitial needle and the surface of ovoid, D) Needle sequence for insertion on the ovoids 
the vertical intrauterine tube at the cervix. This was necessary because the distance of the probe entering the rectum was now shorter and the angle could be adjusted; thus, the scan could be performed vertical to the intrauterine tube. During the guiding process the probe was moved smoothly in the rectum for scanning. Longitudinal and transverse scanning were performed after insertion. To adjust for postoperative patient movement, the position of the patient with the overall applicators was restored. MRI scans (PHILIPS, Amsterdam, Netherlands, 3.0 T) were then performed immediately, with the horizontal position scanned perpendicular to the intrauterine tube $[15,16,17]$.

The probe operator recorded distance measurements for each needle after insertion, with all measurements taken at $1 \mathrm{~cm}$ behind the needle tip. The measuring plane was the cervical section immediately above the ovoids (above the vaginal gas). Using the transverse diameter of the cervix as a baseline, D1 was defined as the distance from the needle to the ventral surface of the cervix, D2 was the distance from the needle to the dorsal surface, D3 was the distance from the needle to the left side, and D4 was the distance from the needle to the right side. In the longitudinal section, the measurement plane clearly showed the inserted needle and uterus, and the depth of insertion (D5) in the vertical direction was calculated as the distance between the needle tip and the surface of the ovoid. D6' was the distance between the needle and the ventral surface of the uterus, while the distance between the needle and the dorsal surface of the uterus was D7' (Figure 2A). The mea-

A

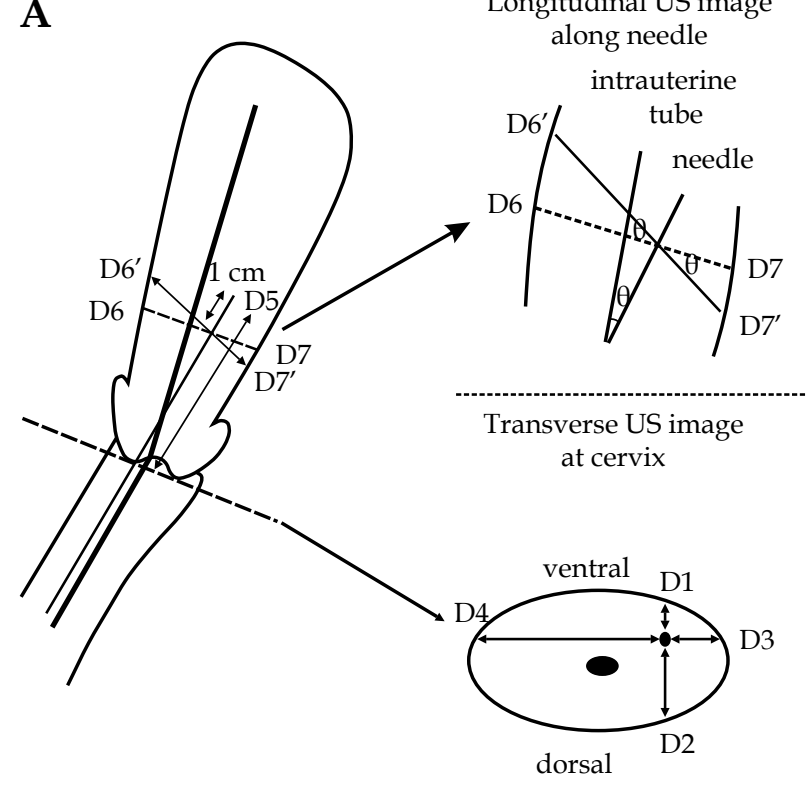

Fig. 2. Measurements of ultrasound data (D1-D7). A) Schematic diagram of ultrasound D measurements, including the measurements of D1-D5, D6' and D7', and the relationships of D6' and D6, and of D7' and D7, B) Ultrasound image of vertical transection of cervix, the measurements of D1-D4, C) Ultrasound image of longitudinal section along inserted needle, the measurements of D5, D6' and D7' surements of the corresponding distances were obtained from the specific ultrasound images (Figure 2B,C).

Based on D6', D7', and $\cos \theta$, the distance between the needle and the ventral surface of the uterus was defined as D6 $=\mathrm{D}^{\prime} \times \cos \theta$, and the distance between the needle and the dorsal surface of the uterus defined as D7 $=$ D7' $\times \cos \theta$. For all measurements, the ultrasonic distance was perpendicular to the needle, while the MRI distance was perpendicular to the intrauterine tube (Figure 2A).

After the MRI examination, the radiologist, who did not know the results of ultrasound-guided insertion process, measured the relevant data for each needle from the MRI images (Figure 3B-D). The cervical section immediately above the ovoids was the measuring plane, with the transverse diameter of the cervix as the baseline. The distances from the needle to the ventral and dorsal cervical surfaces and the left and right sides were designated as M1-M4, respectively. In the sagittal or coronal position, M5 was the distance between the needle tip and the surface of the ovoid. In the transverse section, the distance between the needle and the ventral surface of the uterus along the midpoint of the rectum was M6, while the distance between the needle and the dorsal surface of the uterus along the midpoint of the rectum was M7 (Figure 3A).

\section{Statistical analysis}

Categorical data were calculated as numbers and percentages and continuous data as means \pm standard
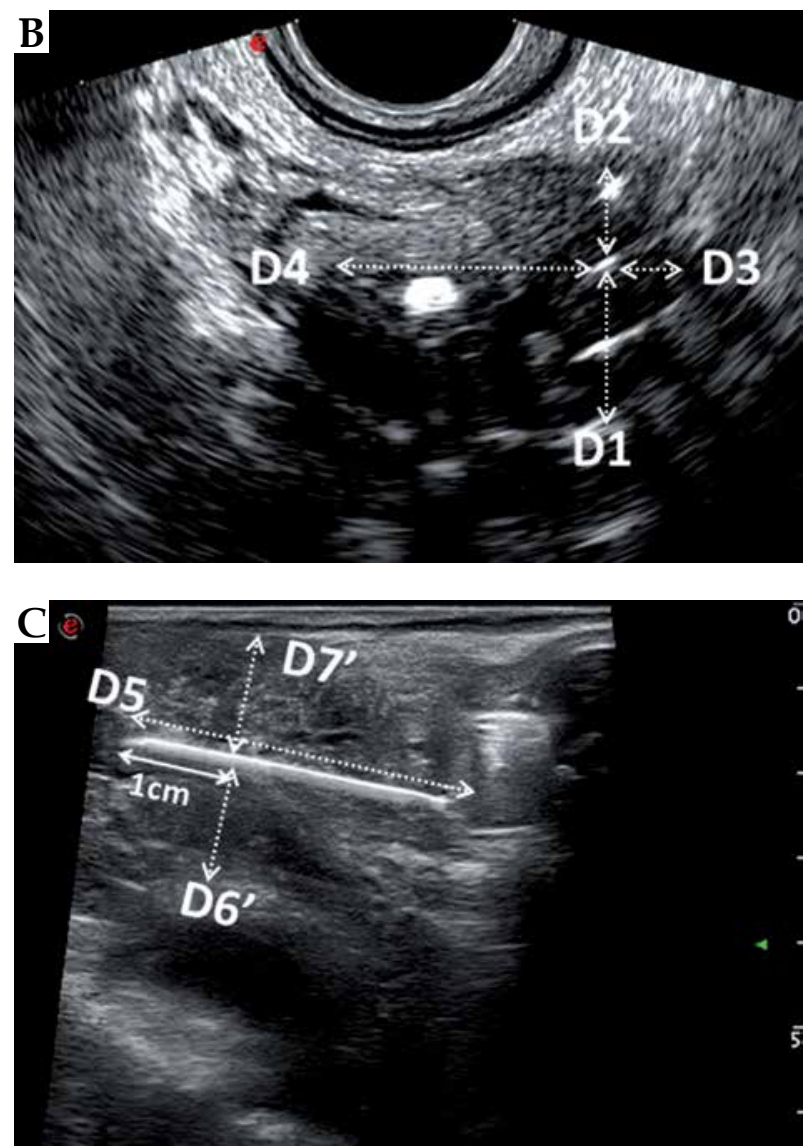
A
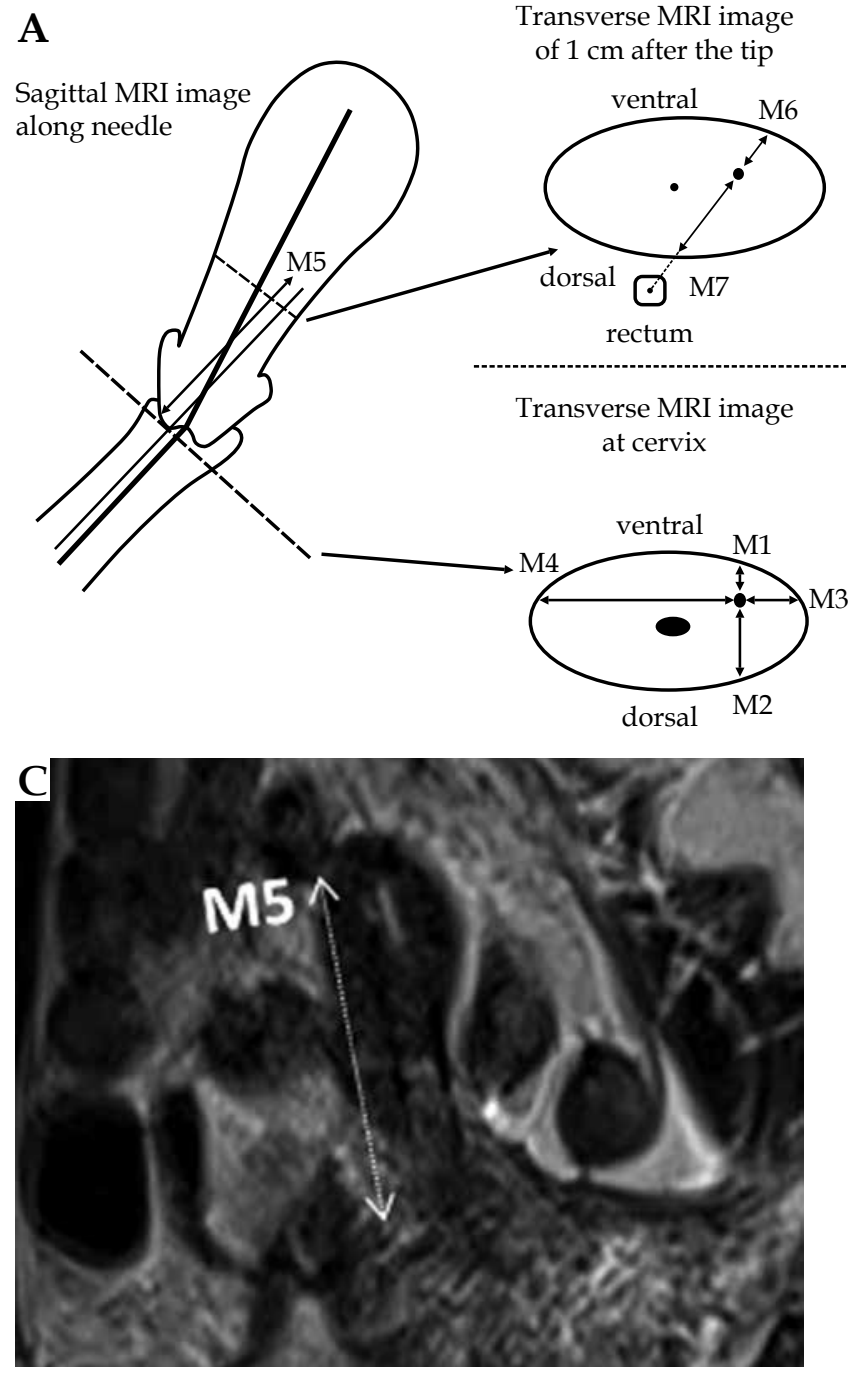
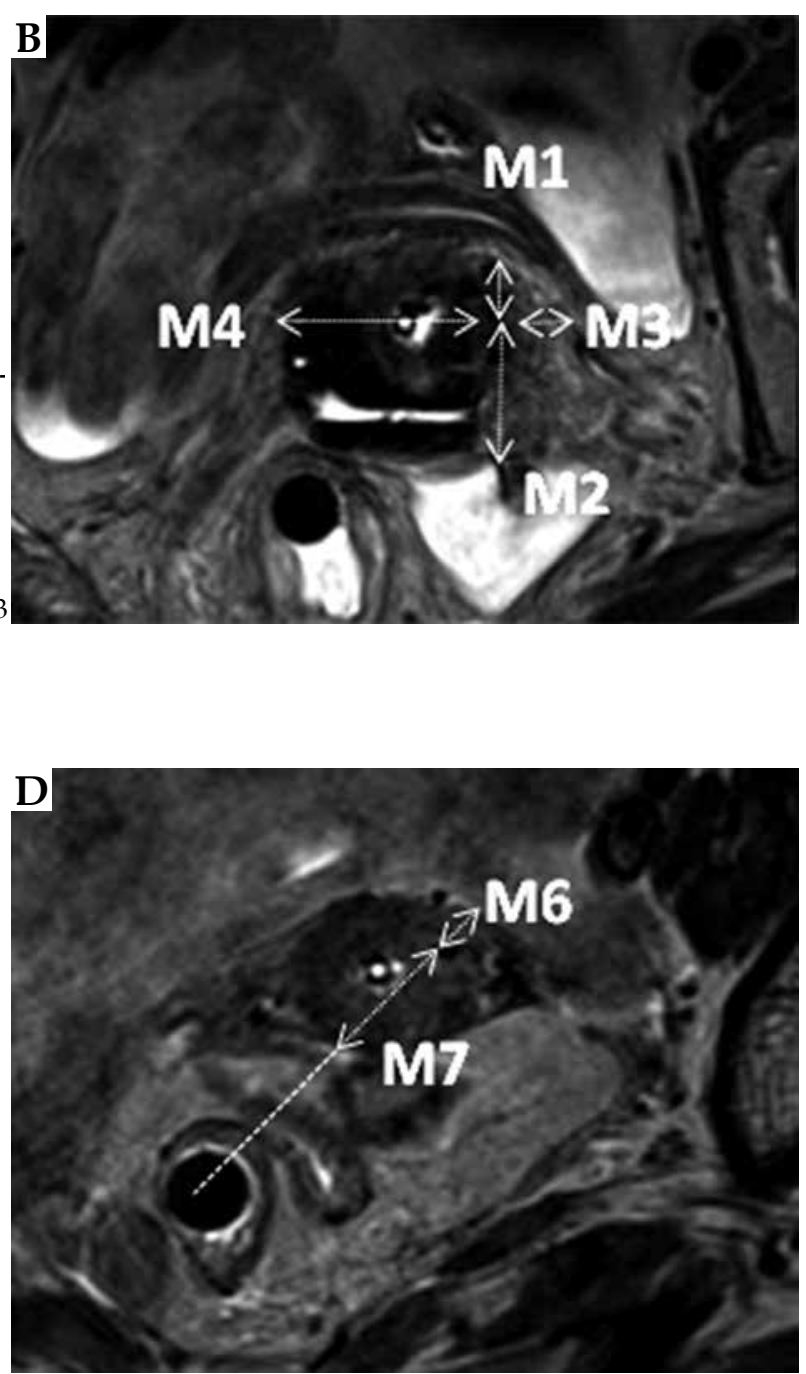

Fig. 3. Measurements of MRI data M1-M7. A) Schematic diagram of MRI data M measurement, the measurements of M1-M7, B) MRI image of cervix transection, the measurements of M1-M4, C) In sagittal/coronal MRI images, the measurement of M5,

D) Cross-sectional MRI image of $1 \mathrm{~cm}$ behind the needle tip, the measurements of M6 and M7

deviations. Differences between the paired ultrasound and MRI measurements were assessed by $t$-tests. The consistency of measurement between the paired MRI (M) and ultrasound (D) data, e.g. M1-D1 and M2-D2, was assessed with intraclass correlation coefficients (ICC). An ICC $>0$ and $<0.10$ was defined as no consistency, $\geq 0.10$ and $<0.40$ was low consistency, 0.41 to 0.60 was moderate consistency, and 0.81 to 1.00 was high consistency [18]. The linear relationships between $\mathrm{M}$ and $\mathrm{D}$ were analysed by regression analysis, with a significance level of $p<0.05$ considered statistically significant. All analyses were performed using SPSS Statistics for Windows, version 22.0 (IBM Corp., Armonk, New York, USA).

\section{Results}

\section{Complications}

Among the 48 patients, 39 (81.3\%) had pain after insertion, six of whom (15.4\%) had severe pain. In all patients, the pain was relieved after symptomatic treatment.
Seven patients $(14.6 \%)$ developed haemorrhages, which were relieved after symptomatic treatments such as local dressing and compression. No patients experienced puncture-related complications such as uterine perforation or damage to the surrounding organs.

\section{Procedural methods}

Based on the pre-treatment plans, 1-10 interstitial needles were required per patient, with insertion depths of $2-5 \mathrm{~cm}$. All patients underwent needle insertion under general anaesthesia, and a total of 337 interstitial needles were inserted. The average depth of insertion was $3.8 \pm 0.7 \mathrm{~cm}$ in ultrasound images. The angles of intrauterine tubes and ovoids for all patients are shown in Table 1.

\section{Differences in $M$ and $D$ measurements}

Analysis of data on the interstitial needles showed that the M, D, and M-D measurements exhibited normal distributions. No significant differences were observed 
between the $\mathrm{M}$ and $\mathrm{D}$ pairs (Table 2). Comparison of the $\mathrm{M}$ and $\mathrm{D}$ measurements showed good consistency; the lowest ICC was observed between M5 and D5 and was in the high category $(\mathrm{ICC}=0.805)$.

\section{Discussion}

Transrectal ultrasound guidance can directly determine the extent of lesions and the location of the area that requires needle insertion. By adjusting the direction and depth of the needle in real time, the accuracy of insertion can be ensured. Furthermore, the probability of inserting into surrounding normal tissue and the incidence of complications, including uterine perforation and bladder or colon damage, can be reduced $[19,20]$. There are few studies comparing the results of ultrasound and MRI after ultrasound-guided insertion [21,22]. MRI has been used to scan the uterus and surrounding tissues, has good resolution in soft tissue tumours, and is the main imaging method used to delineate the target area in brachytherapy, while transrectal ultrasound can be used to scan from the rectum. Both are very good imaging methods, but their scanning methods and mechanisms are different. Theoretically, the measured results of $\mathrm{M}$ and $\mathrm{D}$ should have good concordance. Reassuringly, there were no significant differences between the results obtained by MRI and ultrasound (Table 2). We also found good consistency between $\mathrm{M}$ and $\mathrm{D}$ for all groups, with the ICC ranging between 0.81 and 0.99 (both considered high). These results show that transrectal ultrasound guidance can be used to accurately judge the position of insertion needles, providing important assistance for clinical operation and post-treatment, and a finding consistent with the theoretical speculation and with other studies $[23,24]$.

The determination of the spatial positions of the interstitial needles requires observation from two different planes. In this study, these two planes were: 1) the cervix and 2) $1 \mathrm{~cm}$ behind the needle tip. The reasons are as follows: 1) The cervix is fixed and easy to identify. When transrectal ultrasound is used to scan the vagina horizontally, the vagina becomes filled with gas. When scanning toward the head side, a reduction in gas can be observed, together with the appearance of an annular hypoechoic position, which refers to the cervix. In the MRI horizontal scans, the head side of the ovoids was the cervix;
Table 1. Angles of Utrecht uterine tube and oval body $(n)$

\begin{tabular}{lcc} 
Uterine tube angle & \multicolumn{2}{c}{ Oval body angle } \\
\cline { 2 - 3 } & $15^{\circ}$ & $25^{\circ}$ \\
\hline $15^{\circ}$ & 30 & 0 \\
\hline $30^{\circ}$ & 14 & 1 \\
\hline $45^{\circ}$ & 2 & 1
\end{tabular}

2) The actual insertion depth of each interstitial needle was $1-5 \mathrm{~cm}$, and the observation position was $1 \mathrm{~cm}$ behind the needle tip, which was convenient and easy to identify. In both planes, horizontal MRI scanning was used for observation. Ultrasound of the cervix used the horizontal scan, while the plane $1 \mathrm{~cm}$ behind the needle tip was accurate only in the longitudinal scan. This is because there were no obvious changes in horizontal ultrasound images near the needle tip, which can determine that the cross-section was on the needle body but cannot locate the exact position of the needle. However, longitudinal scanning along the inserted needle showed the full length of the needle and clearly determined its position.

There were no significant differences in $M$ and $D$ among the groups; however, the ICC of M and D in different sections varied. The ICC of D1-D4 with the related MRI measurement data were all $>0.90$. Because the cervix plane is relatively fixed, the lower part is vaginal gas and the gas echo at the cervix plane disappeared; thus, the ultrasonic cross-section could clearly determine the position of the cervix and measure D1-D4. MRI can also determine this plane, and the distances of the needles can be measured to obtain M1-M4. Compression of the rectal probe during ultrasound scan and changes in body position during MRI can affect the consistency of the two sets of data; however, the effects are small, with the results of this study showing high levels of consistency between D1-D4 and M1-M4 [25,26,27].

During insertion, the depth of insertion was determined according to the ultrasound images and the pre-treatment plan. The planned depth of insertion ranged from 2 to $5 \mathrm{~cm}$; the actual insertion depths (D5 and M5) were smaller than planned due to the strength

Table 2. Analysis of $M$ and $D$

\begin{tabular}{lcccccc} 
Groups & $M(\mathrm{~cm})$ & $\mathrm{D}(\mathrm{cm})$ & $|\mathrm{M}-\mathrm{D}|(\mathrm{cm})$ & $t^{*}$ & $p^{*}$ & ICC $^{\#}$ \\
\hline M1-D1 & $1.228 \pm 0.632$ & $1.261 \pm 0.621$ & $0.034 \pm 0.319$ & 1.897 & 0.059 & 0.931 \\
\hline M2-D2 & $1.489 \pm 0.752$ & $1.501 \pm 0.747$ & $0.013 \pm 0.391$ & 0.591 & 0.555 & 0.927 \\
\hline M3-D3 & $2.522 \pm 1.156$ & $2.562 \pm 1.195$ & $0.041 \pm 0.439$ & 1.708 & 0.089 & 0.964 \\
\hline M4-D4 & $1.797 \pm 1.247$ & $1.825 \pm 1.235$ & $0.026 \pm 0.305$ & 1.647 & 0.100 & 0.985 \\
\hline M5-D5 & $3.721 \pm 0.849$ & $3.684 \pm 0.704$ & $0.037 \pm 0.630$ & 1.079 & 0.281 & 0.805 \\
\hline M6-D6 & $0.805 \pm 0.602$ & $0.885 \pm 0.540$ & $0.080 \pm 0.426$ & 1.351 & 0.183 & 0.839 \\
\hline M7-D7 & $1.368 \pm 0.813$ & $1.357 \pm 0.708$ & $0.012 \pm 0.504$ & 0.424 & 0.672 & 0.877
\end{tabular}

${ }^{*}$ is the $t$-value and $p$-value of the paired $t$-test for $M$ and $D$, $\#$ is the intraclass correlation coefficient (ICC) of $M$ and $D$ 

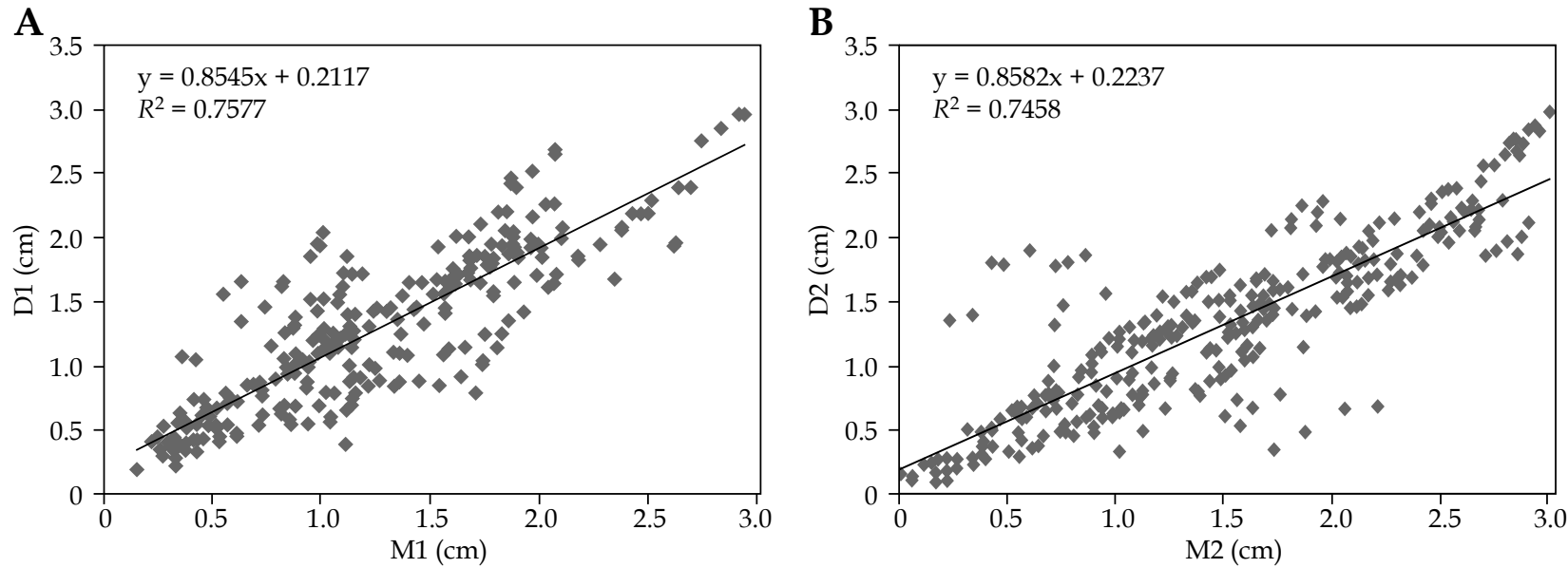

C
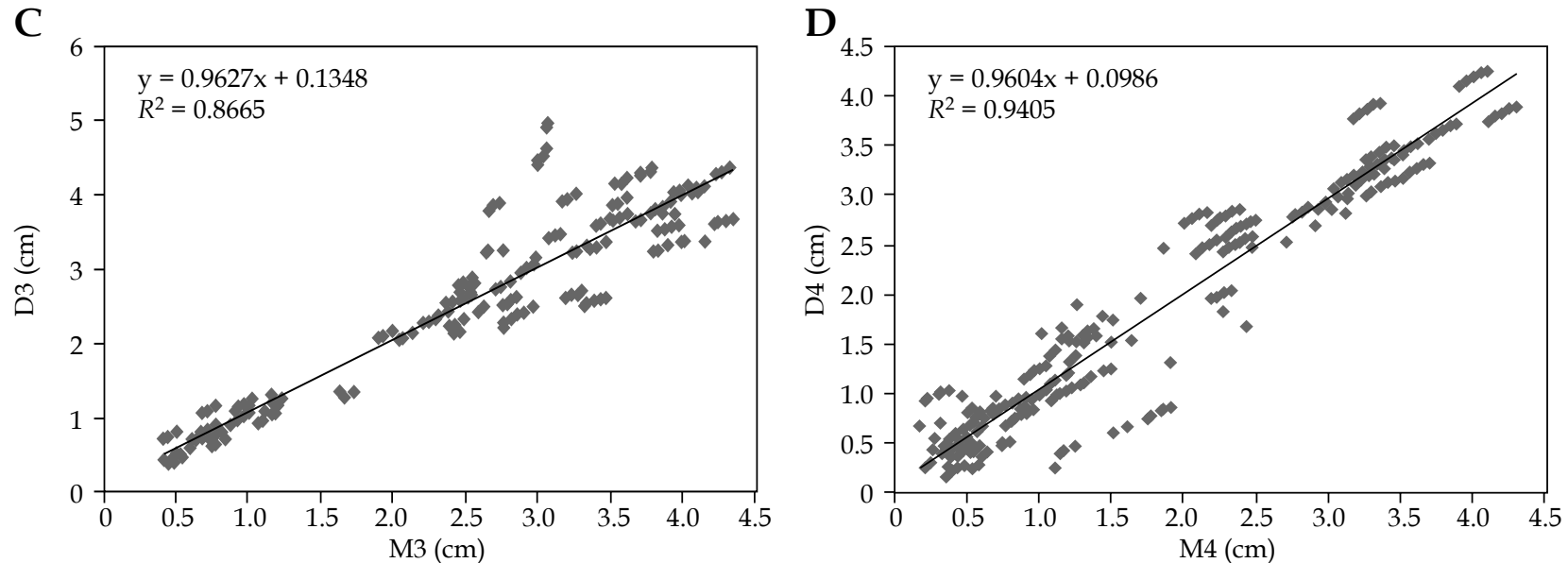

E
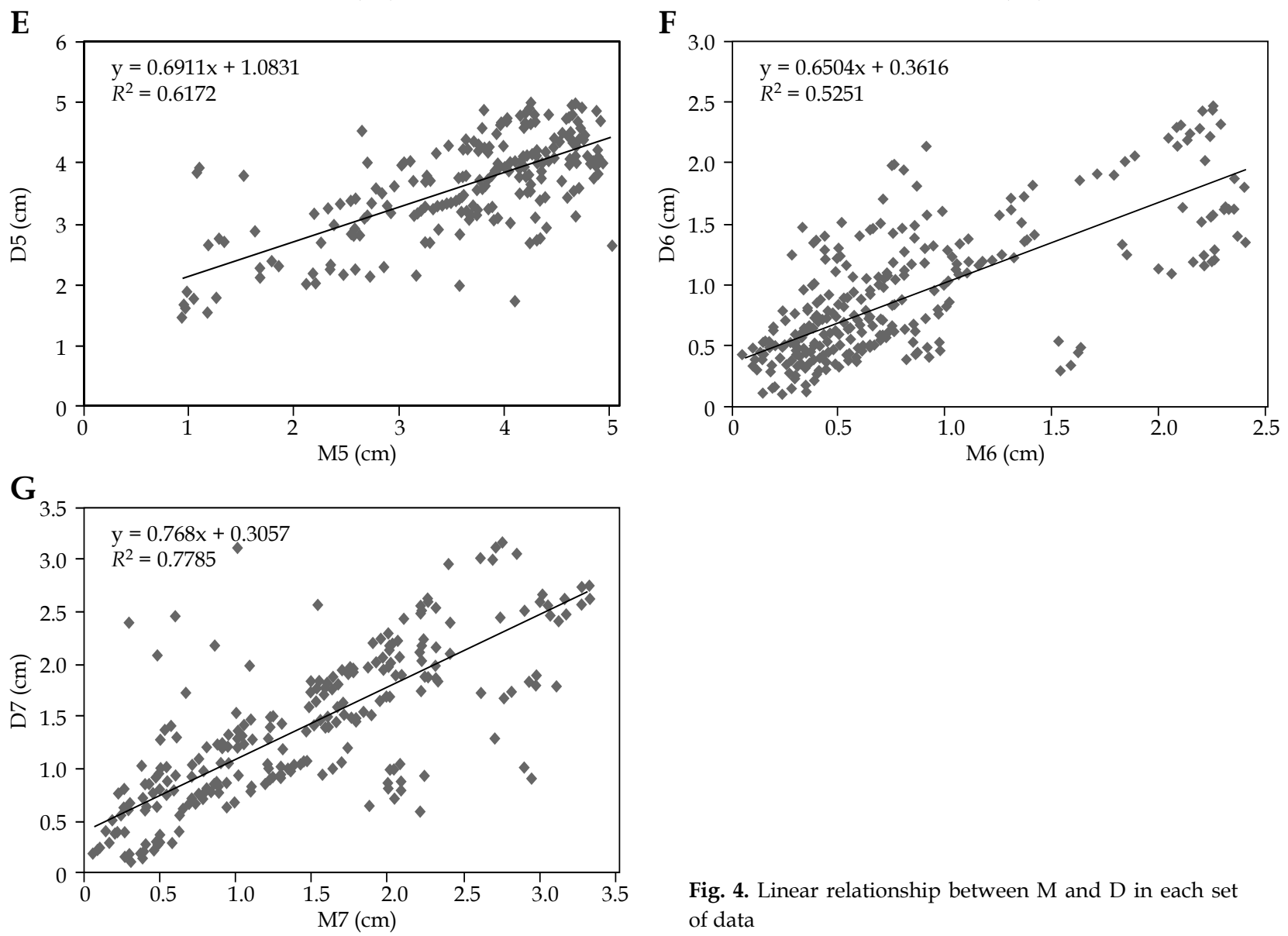

Fig. 4. Linear relationship between $\mathrm{M}$ and $\mathrm{D}$ in each set of data 
of insertion, as well as tissue hardness, elasticity, and degree of fibrosis. In addition, D5 measured by transrectal ultrasound in the longitudinal section was the distance from the needle tip to the surface of the ovoid. There was a certain distance between the inserting point of the needle and the surface of the ovoid, making D5 smaller than the actual insertion depth (Figure 1C). At the same time, the horizontal position of MRI was scanned perpendicular to the intrauterine tube. The interstitial needle might not have been located in the eccentric or coronal position due to being blocked by the tissue, which also made it difficult to measure the actual M5 insertion depth. Therefore, the comparison between D5 and M5 showed a larger deviation and the lowest ICC (0.81).

In this study, the planned depth of each needle was $2-5 \mathrm{~cm}$. In the longitudinal scan, if the probe was parallel to the intrauterine tube, the vertical distance between the probe was the distance vertical to the intrauterine tube. In the actual operation, the probe entered the rectum over a long distance, making it difficult to maintain parallel to the intrauterine tube due to the uterine angle, rectal position, and intestinal gas. However, exploration along the direction of the needle was relatively easy and clearly showed the full length of the needle. The angle $\theta$ between the line D6' to D7' and the line D6 to D7 was equal to the angle of the intrauterine tube minus the angle of the ovoids, D6 $=\mathrm{D}^{\prime} \times \cos \theta, \mathrm{D} 7=\mathrm{D}^{\prime} \times \cos \theta$ (Figures $1 \mathrm{~B}$ and 2A). MRI can identify the cross-section $1 \mathrm{~cm}$ behind the tip, and transrectal ultrasound scans are performed from the centre of the rectum. Therefore, M6, the distance between the needle and the ventral surface of the uterus, and M7, the distance between the needle and the dorsal surface of the uterus, can be measured along the line between the centre of the rectum and the needle. The ICCs between M6 and D6 and between M7 and D7 were high: 0.84 and 0.88 , respectively. Likely reasons that the concordance was not perfect include the following: 1) During intraoperative insertion, the angle, direction, and position of the transrectal ultrasound probe required adjustment, so the rectum might have deviated from its normal position but returned during the MRI; 2) The MRI layer thickness was $0.5 \mathrm{~mm}$; thus, the measurement plane might not always have been accurately positioned $10 \mathrm{~mm}$ behind the needle tip while on an adjacent plane; 3) During actual insertion, the needle may have been displaced due to external forces such as tissue hardness, which may have changed the angle $\theta$.

Based on the measurements between transrectal ultrasound and MRI, this study concluded that transrectal ultrasound and MRI had good concordance in determining the location of the interstitial needle and can play an important role in the layout of needles in a lesion without touching normal surrounding tissues. In addition, ultrasound guidance has the advantages of convenience, consistency, low cost, and no radiation. Due to the limitation of the sample size in the present study, additional studies with large sample sizes are required. In addition, comparative studies are needed of the long-term therapeutic effects, occurrence of complications, treatments using different ultrasound-guided applicators, determination of ultrasound-assisted tumour active areas, and further assessment of the accuracy of target area imaging by ultrasound $[9,10,28,29,30]$.

\section{Conclusions}

In summary, transrectal ultrasound shows good agreement with MRI results in determining the position of the interstitial needle. The images provided accurate information regarding where to insert the needle and how to avoid damage to high-risk areas. Transrectal ultrasound should be considered an appropriate and practical method of needle insertion guidance during interstitial brachytherapy of locally advanced cervical cancer.

\section{Ethics}

This study was conducted in accordance with the declaration of Helsinki. This study was conducted with approval from the Ethics Committee of Jinlin University. Written informed consent was obtained from all participants.

\section{Acknowledgements}

This study was funded by the Bethune Special Research of Science and Technology Department of Jilin Province (20160101079JC); Health Special Fund Project of Jilin Province Finance Department (SCZSY201618).

\section{Disclosure}

The authors report no conflict of interest.

\section{References}

1. Bray F, Ferlay J, Soerjomataram I et al. Global Cancer Statistics 2018: GLOBOCAN estimates of incidence and mortality worldwide for 36 cancers in 185 countries. CA Cancer J Clin 2018; 68: 394-424.

2. Iwata T, Miyauchi A, Suga Y et al. Neoadjuvant chemotherapy for locally advanced cervical cancer. Chin J Cancer Res 2016; 28: 235-240.

3. Karlsson J, Dreifaldt AC, Mordhorst LB et al. Differences in outcome for cervical cancer patients treated with or without brachytherapy. Brachytherapy 2017; 16: 133-140.

4. Crossley N, Tipton C, Meier T et al. The value of hybrid interstitial tandem and ring applicators for organ at risk dose reduction in small volume cervical cancer. J Contemp Brachytherapy 2020; 12: 12-16.

5. Castelnau-Marchand P, Chargari C, Haie-Meder $\mathrm{C}$ et al. Image-guided adaptive brachytherapy in locally advanced cervical cancer: recent advances and perspectives. Curr Opin Oncol 2016; 28: 419-428.

6. Siddanna RP, Chitradurga ART, Thimmaiah $\mathrm{N}$ et al. Magnetic resonance image-based 3D volume interstitial brachytherapy using polyether ether ketone catheters in advanced cervical cancer-a feasibility study. J Contemp Brachytherapy 2019; 11: 307-311.

7. Umesh M, Shivakumar G, Roshni S et al. Indian Brachytherapy society guidelines for radiotherapeutic management of cervical cancer with special emphasis on high-dose-rate brachytherapy. J Contemp Brachytherapy 2019; 11: 293-306.

8. Jean-Guy B, Kundan T, Robyn B et al. The impact of inter-fraction changes for perineal template-based interstitial 
gynecologic brachytherapy implants. J Contemp Brachytherapy 2019; 11: 122-127.

9. Sharma DN, Rath GK, Thulkar S et al. Use of transrectal ultrasound for high dose rate interstitial brachytherapy for patients of carcinoma of uterine cervix. J Gynecol Oncol 2010; 21: 12-17.

10. Van Dyk S, Narayan K, Franzcr et al. Conformal brachytherapy planning for cervical cancer using transabdominal ultrasound. Int J Radiat Oncol Biol Phys 2009; 75: 64-70.

11. Van Dyk S, Bernshaw D. Ultrasound-based conformal planning for gynaecological brachytherapy. J Med Imaging Rad Oncol 2008; 52: 77-84.

12. Tharavichitkul E, Chakrabandhu S, Klunklin P et al. Intermediate-term results of trans-abdominal ultrasound (TAUS)-guided brachytherapy in cervical cancer. Gynecol Oncol 2018; 148: 468-473.

13. van Dyk S, Garth M, Oates A et al. Reproducibility and interoperator reliability of obtaining images and measurements of the cervix and uterus with brachytherapy treatment applicators in situ using transabdominal ultrasound. Brachytherapy 2016; 15: 71-78.

14. Nesvacil N, Schmid MP, Potter R et al. Combining transrectal ultrasound and CT for image-guided adaptive brachytherapy of cervical cancer: Proof of concept. Brachytherapy 2016; 15: 839-844.

15. Viswanathan AN, Erickson B, Gaffney DK et al. Comparison and consensus guidelines for delineation of clinical target volume for CT- and MR-based brachytherapy in locally advanced cervical cancer. Int J Radiat Oncol Biol Phys 2014; 90: 320-328.

16. Pötter R, Federico M, Sturdza A et al. Value of magnetic resonance imaging without or with applicator in place for target definition in cervix cancer brachytherapy. Int J Radiat Oncol Biol Phys 2016; 94: 588-597.

17. Nesvacil N, Pötter R, Sturdza A et al. Adaptive image guided brachytherapy for cervical cancer: a combined MRI-/ CT-planning technique with MRI only at first fraction. Radiother Oncol 2013; 107: 75-81.

18. Shrout PE. Measurement reliability and agreement in psychiatry. Stat Methods Med Res 1998; 7: 301-317.

19. De Brabandere M, Mousa AG, Nulens A et al. Potential of dose optimisation in MRI-based PDR brachytherapy of cervix carcinoma. Radiother Oncol 2008; 88: 217-226.

20. Lindegaard JC, Tanderup K, Nielsen SK et al. MRI-guided 3D optimization significantly improves DVH parameters of pulsed-dose-rate brachytherapy in locally advanced cervical cancer. Int J Radiat Oncol Biol Phys 2008; 71: 756-764.

21. Richart J, Carmona-Meseguerb V, García-Martínezc T et al. Review of strategies for MRI based reconstruction of endocavitary and interstitial applicators in brachytherapy of cervical cancer. Rep Pract Oncol Radiother 2018; 23: 547-561.

22. van Dyk S, Kondalsamy-Chennakesavan S, Schneider M et al. Assessing changes to the brachytherapy target for cervical cancer using a single MRI and serial ultrasound. Brachytherapy 2015; 14: 889-897.

23. Schmid MP, Nesvacil N, Potter R et al. Transrectal ultrasound for image-guided adaptive brachytherapy in cervix cancer - an alternative to MRI for target definition? Radiother Oncol 2016; 120: 467-472.

24. Fischerova D, Cibula D, Stenhova $\mathrm{H}$ et al. Transrectal ultrasound and magnetic resonance imaging in staging of early cervical cancer. Int J Gynecol Cancer 2008; 18: 766-772.

25. Van Dyk S, Garth M, Oates A et al. Reproducibility and interoperator reliability of obtaining images and measurements of the cervix and uterus with brachytherapy treatment applicators in situ using transabdominal ultrasound. Brachytherapy 2016; 15: 71-78.
26. Csutak C, Badea R, Bolboaca SD et al. Multimodal endocavitary ultrasound versus MRI and clinical findings in pre- and post-treatment advanced cervical cancer. Preliminary report. Medical Ultrasonography 2016; 18: 75-81.

27. Van Dyk S, Kondalsamy-Chennakesavan S, Schneider M et al. Comparison of measurements of the uterus and cervix obtained by magnetic resonance and transabdominal ultrasound imaging to identify the brachytherapy target in patients with cervix cancer. Int I Radiat Oncol Biol Phys 2014; 88: 860-865.

28. Mahantshetty U, Khanna N, Swamidas J et al. Trans-abdominal ultrasound (US) and magnetic resonance imaging (MRI) correlation for conformal intracavitary brachytherapy in carcinoma of the uterine cervix. Radiother Oncol 2012; 102: 130-134.

29. Ordeanu C, Pop DC, Badea R et al. Local experience in cervical cancer imaging: comparison in tumour assessment between TRUS and MRI. Rep Pract Oncol Radiother 2015; 20: 223-230.

30. Pinkavova I, Fischerova D, Zikan M et al. Transrectal ultrasound and magnetic resonance imaging in the evaluation of tumor size following neoadjuvant chemotherapy for locally advanced cervical cancer. Ultrasound Obstet Gynecol 2013; 42: 705-712. 